\title{
Hausdorff measure of uniform self-similar fractals
}

\author{
Wolfgang Kreitmeier
}

February 8, 2010

\begin{abstract}
Let $d \geq 1$ be an integer and $E$ a self-similar fractal set, which is the attractor of a uniform contracting iterated function system (UIFS) on $\mathbb{R}^{d}$. Denote by $D$ the Hausdorff dimension, by $\mathcal{H}^{D}(E)$ the Hausdorff measure and by $\operatorname{diam}(E)$ the diameter of $E$. If the UIFS is parametrised by its contracting factor $c$, while the set $\omega$ of fixed points of the UIFS does not depend on $c$, we will show the existence of a positive constant depending only on $\omega$, such that the Hausdorff dimension is smaller than one and $\mathcal{H}^{D}(E)=\operatorname{diam}(E)^{D}$ if $c$ is smaller than this constant. We apply our result to modified versions of various classical fractals. Moreover we present a parametrised UIFS where $\omega$ depends on $c$ and $\mathcal{H}^{D}(E)<\operatorname{diam}(E)^{D}$, if $c$ is small enough.
\end{abstract}

$\operatorname{MSC}(2010): 28 \mathrm{~A} 80,28 \mathrm{~A} 78$

keywords: Self-similar set, Hausdorff measure

\section{Introduction}

Let $d \in \mathbb{N}:=\{1,2, .$.$\} and A \subset \mathbb{R}^{d}$. Let $\varepsilon>0$ and $I \subset \mathbb{N}$. The collection of sets $\left(U_{i}\right)_{i \in I}$ is an $\varepsilon$-cover of $A$, if $A$ is covered by the union of all $U_{i}$ and each set $U_{i}$ does have at most diameter $\varepsilon$, i.e.

$$
\operatorname{diam}\left(U_{i}\right)=\sup \left\{\|x-y\|: x, y \in U_{i}\right\} \leq \varepsilon,
$$


where $\|\cdot\|$ denotes the Euclidean norm. Introduced by Hausdorff [6], for $\alpha \geq 0$, the $\alpha$-Hausdorff measure $\mathcal{H}^{\alpha}(A)$ of $A$ is defined by

$$
\mathcal{H}^{\alpha}(A)=\lim _{\varepsilon \rightarrow 0}\left(\inf \left\{\sum_{i \in I} \operatorname{diam}\left(U_{i}\right)^{\alpha}:\left(U_{i}\right)_{i \in I} \text { is an } \varepsilon \text {-cover of } A\right\}\right) .
$$

It is easy to check, that $\mathcal{H}^{\alpha}(A)$ is non-increasing in $\alpha$ and that $\mathcal{H}^{\beta}(A)>0$ for a $\beta>0$ implies $\mathcal{H}^{\alpha}(A)=\infty$ for all $0 \leq \alpha<\beta$. Therefore, the Hausdorff dimension of $A$ is well-defined as

$$
\operatorname{dim}_{H}(A)=\sup \left\{\beta \geq 0: \mathcal{H}^{\beta}(F)=\infty\right\}=\inf \left\{\beta \geq 0: \mathcal{H}^{\beta}(F)=0\right\}
$$

Although the Hausdorff dimension was computed for a large class of fractal sets (cf. [8, 9, 16] and the references therein), the Hausdorff measure has been calculated exactly only for a few fractals so far. Exact results for selfsimilar fractal sets in one dimension were derived by several authors (cf. [1, 14, 15, 23] and the references therein). Also in the non-self-similar case exact values were calculated under certain conditions (cf. [18]). But for higher dimensional fractals little is known about the exact value of their Hausdorff measure.

The exact value of the Hausdorff measure for the classical Sierpinski gasket is still unknown, but can be approximated arbitrarily well (cf. [2, 10]). For a class of generalized Sierpinski gaskets and Sierpinski sponges, the Hausdorff measure was calculated exactly (cf. [5, 25, 26]). Also the Hausdorff measure of the Sierpinski carpet was investigated by several authors (cf. [3, 4, 21]). Recently, the exact value of the Hausdorff measure for a class of regular homogeneous Moran sets with Hausdorff dimension greater than one was calculated (cf. [19]). Moreover, an effective method for computing the exact value of the Hausdorff measure of a class of self-similar fractal sets has been provided (cf. [22]). Nevertheless, beside of these explicit calculations for special examples, a general approach in calculating the exact value of the Hausdorff measure of a (self-similar) fractal is still missing (cf. [24]). The reader is also referred to Zhou et.al. [27] for another survey of open problems in fractal geometry.

In this paper we consider uniformly contracting iterated function systems (UIFS) and their related fractal attractor set $E$. If the UIFS is parametrised by its contracting factor $c$, but the set $\omega$ of fixed points of the UIFS is independent of $c$, we will show (cf. theorem 2.7), that the Hausdorff measure $\mathcal{H}^{D}(E)$ 
of $E$ with Hausdorff dimension $D$ equals $\operatorname{diam}(E)^{D}$, if $c$ is smaller than a constant, which depends only on $\omega$. If the set $\omega$ satisfies a special condition, we can determine a lower bound for this constant (cf. theorem 2.5, remark 2.6). In this respect we give an answer to problem 8 in [24]. Moreover we present a parametrised UIFS, where $\omega$ depends on $c$ and $\mathcal{H}^{D}(E)<\operatorname{diam}(E)^{D}$, if $c$ is small enough (cf. example 4.6). Our approach is based on an inverse density characterization of $\mathcal{H}^{D}(E)$, developed by several authors (cf. [1, 12, 15]).

\section{Self-similar sets and their Hausdorff mea- sure}

Let $N \in \mathbb{N}$ with $N \geq 2$. Let $\omega=\left\{x_{1}, . ., x_{N}\right\} \subset \mathbb{R}^{d}$ be a set consisting of $N$ different points, i.e. $\operatorname{card}(\omega)=N$ if card denotes cardinality.

Recall $\|\cdot\|$ as the Euclidean norm on $\mathbb{R}^{d}$ and let $\left(S_{1}, . ., S_{N}\right)$ be a system of contracting similitudes on $\mathbb{R}^{d}$ with $\omega$ as the set of its fixed points and $\left(c_{1}, . ., c_{N}\right)$ as its contracting ratios, i.e. $S_{i}\left(x_{i}\right)=x_{i}$ and $\left\|S_{i}(x)-S_{i}(y)\right\|=$ $c_{i}\|x-y\|$ for every $i \in\{1, . ., N\}$ and $x, y \in \mathbb{R}^{d}$. We call $\left(S_{1}, . ., S_{N}\right)$ an iterated function system (IFS). It is well-known (cf. [11]), that every IFS generates a unique nonempty compact set $E \subset \mathbb{R}^{d}$, which is characterised by

$$
E=\bigcup_{i=1}^{N} S_{i}(E) .
$$

The set $E$ is often called invariant attractor or attractor set. Moreover, $\omega$ is a subset of $E$. Throughout the paper we will assume w.l.o.g that the span of $E$ is of dimension $d$. To be able to investigate the Hausdorff measure of $E$ we need the following well-known separation condition.

(OSC) there exists a bounded nonempty open set $O \subset \mathbb{R}^{d}$ such that $S_{i}(O) \subset O$ for every $i \in\{1, . ., N\}$ and $S_{i}(O) \cap S_{j}(O)=\emptyset$ for every $i, j \in\{1, . ., N\}$ with $i \neq j$.

Condition (OSC) is called open set condition in the literature. Another important separation condition is the so-called strong separation condition (SSC), which is satisfied, if $S_{i}(E) \cap S_{j}(E)=\emptyset$ for every $i, j \in\{1, . ., N\}$ with $i \neq j$. It is easy to prove (cf. [11], example 5.2.(2)(a)), that (SSC) implies

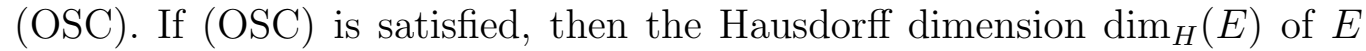


equals the similarity dimension $D$ of $E$ (cf. [11]), which is defined as the unique real number satisfying

$$
\sum_{i=1}^{N} c_{i}^{D}=1
$$

It is also well known (cf. [11, 17]), that (OSC) implies $0<\mathcal{H}^{D}(E)<\infty$. If (SSC) holds, let

$$
h=\min \left\{\operatorname{dist}\left(S_{i}(E), S_{j}(E)\right): i, j \in\{1, . ., N\}, i \neq j\right\}>0 .
$$

Moreover we denote

$$
s=\min _{i \in\{1, . ., N\}} c_{i} \quad \text { resp. } \quad t=\max _{i \in\{1, . ., N\}} c_{i} .
$$

For any $k \in \mathbb{N}$, nonempty set $\Gamma \subset\{1, . ., N\}^{k}$ and $\sigma=\left(\sigma_{1}, . ., \sigma_{k}\right) \in \Gamma$ we define $c_{\sigma}=c_{\sigma_{1}} \cdot \ldots \cdot c_{\sigma_{k}}$ resp. $S_{\sigma}=S_{\sigma_{1}} \circ \cdots \circ S_{\sigma_{k}}$ and $E_{\Gamma}=\bigcup_{\sigma \in \Gamma} S_{\sigma}(E)$.

Several authors (cf. [1, 12, 15]) introduced a characterization of $\mathcal{H}^{D}(E)$ in terms of an inverse density. In this paper we rely on the following results.

Theorem 2.1 ([15]).

(a) If $\operatorname{dim}_{H}(E) \leq t$, then $\mathcal{H}^{t}(E) \leq \operatorname{diam}(E)^{t}$.

(b) If the IFS satisfies condition (OSC), then

$$
0<\mathcal{H}^{D}(E)=\inf \left\{\frac{\operatorname{diam}\left(E_{\Gamma}\right)^{D}}{\sum_{\sigma \in \Gamma} c_{\sigma}^{D}} ; \emptyset \neq \Gamma \subset\{1, . ., N\}^{k}, k \in \mathbb{N}\right\} .
$$

(c) If the IFS satisfies condition $(S S C)$ and $D<1$, then

$$
0<h^{D} \leq \mathcal{H}^{D}(E)=\min \left\{\frac{\operatorname{diam}\left(E_{\Gamma}\right)^{D}}{\sum_{\sigma \in \Gamma} c_{\sigma}^{D}} ; \emptyset \neq \Gamma \subset\{1, . ., N\}^{k_{0}}\right\},
$$

with

$$
k_{0}=\min \left\{k \in \mathbb{N}: \operatorname{diam}(E) \leq h \cdot s \cdot t^{k(D-1)}\right\} .
$$

Remark 2.2. To be more precise we mention that assertion (a) follows from [15, corollary 4.2]. From [15, theorem 6.2] we obtain part (b). If condition (SSC) is satisfied, [15, corollary 6.3] yields $h^{D} \leq \mathcal{H}^{D}(E)$. If, additionally, $D<1$, then [15, corollary 6.4] proves the remaining part of assertion (c). 
For any $k \in \mathbb{N}$ and $\Gamma \subset\{1, . ., N\}^{k}$ let

$$
I_{\Gamma}=\left\{i \in\{1, . ., N\}: E_{\Gamma} \cap S_{i}(E) \neq \emptyset\right\} .
$$

If condition (SSC) and $D<1$ holds, we define with $k_{0}$ from (3) the set

$$
\mathcal{E}=\left\{E_{\Gamma}: \Gamma \subset\{1, . ., N\}^{k_{0}}, \operatorname{card}\left(I_{\Gamma}\right)>1 \text { and } \mathcal{H}^{D}(E)=\frac{\operatorname{diam}\left(E_{\Gamma}\right)^{D}}{\sum_{\sigma \in \Gamma} c_{\sigma}^{D}}\right\} .
$$

Remark 2.3. Assume that all conditions of theorem 2.1 (c) are satisfied and let $\Gamma \subset\{1, . ., N\}^{k_{0}}$ with $\mathcal{H}^{D}(E)=\frac{\operatorname{diam}\left(E_{\Gamma}\right)^{D}}{\sum_{\sigma \in \Gamma} c_{\sigma}^{D}}$. Assume further, that $\operatorname{card}\left(I_{\Gamma}\right)=1$. Let $j \in\{1, . ., N\}$ with $I_{\Gamma}=\{j\}$. Using (1) we have $E_{\Gamma} \subset$ $S_{j}(E)$, resp. $\sigma_{1}=j$ for every $\left(\sigma_{1}, . ., \sigma_{k_{0}}\right) \in \Gamma$. If $k_{0} \geq 2$, then define $\Gamma^{\prime}=\left\{\left(\sigma_{2}, . ., \sigma_{k_{0}}\right):\left(\sigma_{1}, . ., \sigma_{k_{0}}\right) \in \Gamma\right\}$. If $k_{0}=1$, let $\Gamma^{\prime}=\{1, . ., N\}$. Again by (1) we obtain $E_{\Gamma^{\prime}}=S_{j}^{-1}\left(E_{\Gamma}\right)$ and

$$
\frac{\operatorname{diam}\left(E_{\Gamma}\right)^{D}}{\sum_{\sigma \in \Gamma} c_{\sigma}^{D}}=\frac{c_{j}^{D} \operatorname{diam}\left(S_{j}^{-1}\left(E_{\Gamma}\right)\right)^{D}}{\sum_{\sigma \in \Gamma} c_{\sigma}^{D}}=\frac{\operatorname{diam}\left(E_{\Gamma^{\prime}}\right)^{D}}{\sum_{\sigma \in \Gamma^{\prime}} c_{\sigma}^{D}} .
$$

Using (1) we deduce $\Gamma^{\prime} \subset\{1, . ., N\}^{\max \left(1, k_{0}-1\right)} \subset\{1, . ., N\}^{k_{0}}$. If necessary, we can repeat this procedure ('blow-up principle' cf. [1, 12]) until card $\left(I_{\Gamma^{\prime}}\right)>1$. Thus we can assume w.l.o.g. that $\operatorname{card}\left(I_{\Gamma}\right)>1$, resp. $E_{\Gamma} \in \mathcal{E}$. Especially $\mathcal{E}$ is a nonempty set.

Remark 2.4. Marion (cf. [15, theorem 7.1]) has shown additionally, that $k_{0}$ in theorem 2.1 (c) can be set equal to 1, if the IFS is one-dimensional $(d=1)$. Ayer and Strichartz (cf. [1, theorem 4.2]) have also shown this result for one-dimensional IFS by an alternative proof. Both proofs depend on one-dimensionality at some key places and cannot be generalized into higher dimensions. If the $(S S C)$ is satisfied (but not necessarily $D<1$ ), then Llorente and Morán ([12, theorem 3.3 (i)]) have proved that a compact non-empty convex set $A \subset \mathbb{R}^{d}$ with $h \leq \operatorname{diam}(A) \leq \operatorname{diam}(E)$ exists, such that

$$
\mathcal{H}^{D}(E)=\frac{\operatorname{diam}(A)^{D}}{\mu(A)}
$$

if $\mu$ denotes the normalized Hausdorff measure on E. One may conjecture, that under these assumptions also a $k<\infty$ and $\Gamma \subset\{1, . ., N\}^{k}$ exists, such that $\mathcal{H}^{D}(E)=\frac{\operatorname{diam}\left(E_{\Gamma}\right)^{D}}{\sum_{\sigma \in \Gamma} c_{\sigma}^{D}}$. Unfortunately this is wrong in general for $D>1$, see e.g. [7]. 
To be able to state the first main result of this paper we need some further technical notations, which will be motivated in more detail in section 3 when we are proving our results. Let $\emptyset \neq I \subset\{1, . ., N\}$ and $\omega_{I}=\left\{x_{i}: i \in I\right\}$. If $N \geq 3$ let

$$
\Delta=\max \left\{\left(\frac{\operatorname{diam}(\omega)}{\operatorname{diam}\left(\omega_{I}\right)}\right)^{D} \frac{\operatorname{card}(I)}{N}: I \subset\{1, . ., N\}, 2 \leq \operatorname{card}(I)<N\right\} .
$$

With $d_{\min }(\omega)=\min \{\|x-y\|: x, y \in \omega, x \neq y\}$ we define

$$
c_{0}= \begin{cases}\min \left(\left(2 N^{2}\right)^{-1}, \frac{1}{4} \frac{d_{\min }(\omega)}{\operatorname{diam}(\omega)}(1-\Delta)\right) & , \text { if } N \geq 3 \\ \frac{1}{8} & , \text { if } N=2 .\end{cases}
$$

If the contracting factors of the IFS are all equal, then we call the IFS a uniform contracting iterated function system (UIFS). The Hausdorff measure of the invariant attractor of a UIFS can be determined if the contracting factor $c$ is smaller than $c_{0}$. This is stated in our first main result.

Theorem 2.5. Consider a UIFS with contracting factor $c$ and fixpoint set $\omega$. In case of $N \geq 3$, assume that $\Delta<1$. If $c$ is smaller than $c_{0}$, then condition $(S S C)$ and $D<1$ is satisfied. Moreover, $\mathcal{H}^{D}(E)=\operatorname{diam}(E)^{D}$ and the set $\mathcal{E}$ consists only of the set E.

The examples 4.3 and 4.5 in section 4 are demonstrating the applicability of theorem 2.5.

Remark 2.6. All quantities appearing on the right side of (7) are invariant under a translation and/or rotation of the whole UIFS. Note, that $\Delta$ depends on the contracting factor $c$. Due to $D<1$ we obtain

$$
\Delta \leq \Delta^{\prime}=\max \left\{\frac{\operatorname{diam}(\omega)}{\operatorname{diam}\left(\omega_{I}\right)} \frac{\operatorname{card}(I)}{N}: I \subset\{1, . ., N\}, 2 \leq \operatorname{card}(I)<N\right\} .
$$

Thus, $\Delta<1$ is satisfied, if $\Delta^{\prime}<1$, which depends only on $\omega$ and not on the contracting factor $c$. Hence, $c_{0}$ depends only on $\omega$ if we substitute $\Delta$ by $\Delta^{\prime}$.

Let us call a UIFS parametrised, if for every $i \in\{1, \ldots, N\}$ the mapping $S_{i}$ is parametrised by the unique contracting factor, i.e. $S_{i}=S_{i, c}$ depends on the contracting factor $c$. Our second main result states as follows. 
Theorem 2.7. For every parametrised UIFS where the set $\omega$ of fixed points does not depend on the contracting factor $c$, there exists a positive constant, depending only on $\omega$, such that for every contracting factor $c$ smaller than this constant

(a) the UIFS satisfies condition (SSC) and $D=D(c)<1$,

(b) $\mathcal{H}^{D}(E)=\operatorname{diam}(E)^{D}$ and

(c) $\mathcal{E}$ consists only of the set $E$.

Example 4.8 demonstrates the applicability of theorem 2.7. For applications, an explicit determination of the constant in theorem 2.7 would be preferable. This is provided by theorem 2.5 under further restrictions on the UIFS. It is natural to ask, if the assertions of theorem 2.7 remain valid, if the set $\omega$ of fixed points is no longer independent from the contracting factor $c$, i.e. if we only require that $c$ is small enough. Example 4.6 gives a negative answer.

\section{Proof of the main result}

For any $x \in \mathbb{R}^{d}$ and $r>0$ we denote $B(x, r)=\left\{z \in \mathbb{R}^{d}:\|z-x\| \leq r\right\}$ as the closed ball around $x$ with radius $r$. Recall

$$
d_{\min }(\omega)=\min \{\|x-y\|: x, y \in \omega, x \neq y\} .
$$

For any $k \geq 2$ and $\tau=\left(\tau_{1}, . ., \tau_{k}\right) \in\{1, . ., N\}^{k}$ we denote $\tau^{-}=\left(\tau_{1}, . ., \tau_{k-1}\right)$. First let us prove a criterion, ensuring that condition (SSC) holds for a UIFS with contracting parameter $c$, attractor $E$ and fixpoint set $\omega$.

\section{Lemma 3.1.}

(a) If $c<\frac{1}{2}$, then $\operatorname{diam}(E)<\frac{\operatorname{diam}(\omega)}{1-2 c}$.

(b) If $c<\frac{d_{\min }(\omega)}{4 \operatorname{diam}(\omega)}$, then $(S S C)$ holds.

Proof. (a) Clearly, $x_{i} \in S_{i}(E)$ for every $i \in\{1, . ., N\}$. Using equation (1) and the compactness of $E$ we have $k, l \in\{1, . ., N\}$ and points $x \in S_{k}(E)$ resp. $y \in S_{l}(E)$ with

$$
\begin{aligned}
\operatorname{diam}(E)=\|x-y\| & \leq\left\|x-x_{k}\right\|+\left\|x_{k}-x_{l}\right\|+\left\|x_{l}-y\right\| \\
& \leq \operatorname{diam}\left(S_{k}(E)\right)+\operatorname{diam}(\omega)+\operatorname{diam}\left(S_{l}(E)\right) \\
& =2 c \operatorname{diam}(E)+\operatorname{diam}(\omega) .
\end{aligned}
$$


With $c<\frac{1}{2}$ we deduce

$$
\operatorname{diam}(E) \leq \frac{\operatorname{diam}(\omega)}{1-2 c}
$$

(b) Combining inequality $(8)$ and $c<\frac{d_{\min }(\omega)}{4 \operatorname{diam}(\omega)}<\frac{1}{2}$ we obtain

$$
\begin{aligned}
2 c \operatorname{diam}(E) & \leq \frac{2 c}{1-2 c} \operatorname{diam}(\omega) \\
& <\frac{d_{\min }(\omega)}{2 \operatorname{diam}(\omega)-d_{\min }(\omega)} \operatorname{diam}(\omega) \\
& \leq d_{\min }(\omega) .
\end{aligned}
$$

Thus we get $S_{i}(E) \cap S_{j}(E) \subset B\left(x_{i}, c \operatorname{diam}(E)\right) \cap B\left(x_{j}, c \operatorname{diam}(E)\right)=\emptyset$ for every $i, j \in\{1, \ldots, N\}$ with $i \neq j$.

We continue with a technical result, which is necessary for the proof of theorem 2.7 resp. theorem 2.5 below.

Lemma 3.2. Let $c<\left(2 N^{2}\right)^{-1}$ and $k \in \mathbb{N}$. Then $\frac{\left(1-2 c^{k}\right)^{D}}{1-c^{(k+1) D}}>1$.

Proof. Clearly $0<D=\frac{-\log (N)}{\log (c)}<1$. Thus, the assertion is true, if

$$
1-2 c^{k}>\left(1-c^{-(k+1) \frac{\log (N)}{\log (c)}}\right)^{\frac{-\log (c)}{\log (N)}}=\left(1-N^{-(k+1)}\right)^{\frac{-\log (c)}{\log (N)}} .
$$

Due to $c<\left(2 N^{2}\right)^{-1}$ one gets $c^{k}<\left(2 N^{(k+1)}\right)^{-1}$ for every $k \in \mathbb{N}$. Thus we deduce $1-2 c^{k}>\left(1-N^{-(k+1)}\right)>\left(1-N^{-(k+1)}\right)^{\frac{-\log (c)}{\log (N)}}$, showing that 96 is true.

As the next step we will prove the assertions of theorem 2.5. Recall the definition (7) of $c_{0}$ and the definition (6) of $\Delta$. Theorem 2.7(c) states that $\mathcal{H}^{D}=\operatorname{diam}(E)^{D}$ if and only if

$$
\operatorname{diam}(E)^{D} \sum_{\sigma \in \Gamma} c_{\sigma}^{D} \leq \operatorname{diam}\left(E_{\Gamma}\right)^{D}
$$

for every nonempty $\Gamma \subset\{1, \ldots, N\}^{k_{0}}$. If $c$ becomes small and if we make the restriction, that $\Gamma \subset\{1, \ldots, N\}$, the left hand side of $(10)$ is approximated by $\operatorname{diam}(\omega)^{D} \frac{\operatorname{card}(\Gamma)}{N}$. Recall $\omega_{\Gamma}=\left\{x_{i}: i \in \Gamma\right\}$. For the right hand side of 10 the value $\operatorname{diam}\left(\omega_{\Gamma}\right)^{D}$ is a good approximation for small $c$. The following proof of theorem 2.5 will rely on these approximation values. To this end we work with $\Delta$ as defined in (6) resp. $c_{0}$ defined in (7). 
Proof of theorem 2.5.

Consider a UIFS with $\Delta<1$ if $N \geq 3$ and contracting factor $c<c_{0}$. Due to $c<\frac{1}{N}$ we deduce $D<1$. Lemma 3.1 (b) ensures, that also condition (SSC) holds for this UIFS. Fix $k_{0}$ as in $\left(\sqrt[3]{3)}\right.$ and let $\Gamma \subset\{1, \ldots, N\}^{k_{0}}$ with

$$
\mathcal{H}^{D}(E)=\frac{\operatorname{diam}\left(E_{\Gamma}\right)^{D}}{\sum_{\sigma \in \Gamma} c_{\sigma}^{D}} \leq \operatorname{diam}(E)^{D}
$$

according to theorem 2.1 (a)/(c). Now assume, that $\operatorname{diam}\left(E_{\Gamma}\right)=\operatorname{diam}(E)$. Using (1) an induction argument shows $E_{\{1, . ., N\}^{k_{0}}}=E$. Also by induction and (2) we deduce $\sum_{\sigma \in\{1, . ., N\}^{k_{0}}} c_{\sigma}^{D}=1$. If $\Gamma \neq\{1, . ., N\}^{k_{0}}$, then we obtain

$$
\mathcal{H}^{D}(E) \leq \operatorname{diam}(E)^{D}=\frac{\operatorname{diam}\left(E_{\{1, . ., N\}^{k_{0}}}\right)^{D}}{\sum_{\sigma \in\{1, . ., N\}^{k_{0}}} c_{\sigma}^{D}}<\frac{\operatorname{diam}\left(E_{\Gamma}\right)^{D}}{\sum_{\sigma \in \Gamma} c_{\sigma}^{D}}
$$

which is a contradiction. Hence $\Gamma=\{1, . ., N\}^{k_{0}}$ and $\mathcal{E}=\{E\}$ resp. $\mathcal{H}^{D}(E)=$ $\operatorname{diam}(E)^{D}$. It remains to prove, that $\operatorname{diam}\left(E_{\Gamma}\right)<\operatorname{diam}(E)$ cannot happen. We proceed indirectly. Assume that

$$
\operatorname{diam}\left(E_{\Gamma}\right)<\operatorname{diam}(E) .
$$

Recall $I_{\Gamma}$ defined in (4). According to remark 2.3 we can assume w.l.o.g. that $\operatorname{card}\left(I_{\Gamma}\right) \geq 2$.

Case 1. $\{1, . ., N\} \backslash I_{\Gamma} \neq \emptyset$.

Using (2) we get

$$
\sum_{\sigma \in \Gamma} c_{\sigma}^{D} \leq \sum_{i \in I_{\Gamma}} c^{D}=\frac{\operatorname{card}\left(I_{\Gamma}\right)}{N}
$$

By the definition of $I_{\Gamma}$ we deduce

$$
\begin{aligned}
\operatorname{diam}\left(E_{\Gamma}\right) & \geq \operatorname{diam}\left(\bigcup_{i \in I_{\Gamma}} S_{i}(E)\right)-2 \max _{i \in I_{\Gamma}} \operatorname{diam}\left(S_{i}(E)\right) \\
& =\operatorname{diam}\left(\bigcup_{i \in I_{\Gamma}} S_{i}(E)\right)-2 c \operatorname{diam}(E) \\
& \geq \operatorname{diam}\left(\omega_{I_{\Gamma}}\right)-2 c \operatorname{diam}(E) .
\end{aligned}
$$

Lemma 3.1 (a) implies

$$
\operatorname{diam}\left(E_{\Gamma}\right) \geq \operatorname{diam}\left(\omega_{I_{\Gamma}}\right)-2 c \frac{\operatorname{diam}(\omega)}{1-2 c} .
$$


The combination of inequality (14) and (13) yields

$$
\begin{aligned}
\frac{\operatorname{diam}\left(E_{\Gamma}\right)^{D}}{\sum_{\sigma \in \Gamma} c_{\sigma}^{D}} & \geq \frac{\left(\operatorname{diam}\left(\omega_{I_{\Gamma}}\right)-2 c \frac{\operatorname{diam}(\omega)}{1-2 c}\right)^{D}}{\frac{\operatorname{card}\left(I_{\Gamma}\right)}{N}} \\
& =\frac{\operatorname{diam}\left(\omega_{I_{\Gamma}}\right)^{D}}{\frac{\operatorname{card}\left(I_{\Gamma}\right)}{N}}\left(1-\frac{2 c}{1-2 c} \frac{\operatorname{diam}(\omega)}{\operatorname{diam}\left(\omega_{I_{\Gamma}}\right)}\right)^{D} \\
& \geq \frac{\operatorname{diam}(\omega)^{D}}{\Delta(1-2 c)^{D}}\left(1-2 c-2 c \frac{\operatorname{diam}(\omega)}{d_{\min }(\omega)}\right)^{D} .
\end{aligned}
$$

With the definition of $c_{0}$ in (7) we deduce

$$
\left(1-2 c-2 c \frac{\operatorname{diam}(\omega)}{d_{\min }(\omega)}\right) \geq\left(1-4 c \frac{\operatorname{diam}(\omega)}{d_{\min }(\omega)}\right)>\Delta .
$$

Using relation (16) and lemma 3.1 (a) we obtain from inequality (15) that

$$
\frac{\operatorname{diam}\left(E_{\Gamma}\right)^{D}}{\sum_{\sigma \in \Gamma} c_{\sigma}^{D}} \geq \operatorname{diam}(E)^{D} \Delta^{D-1}>\operatorname{diam}(E)^{D} .
$$

The last inequality follows from $0<D<1$ and $0<\Delta<1$. But (17) contradicts (11).

Case 2. $I_{\Gamma}=\{1, . ., N\}$.

Let $I=\left\{i \in I_{\Gamma}: S_{i}(E) \subset E_{\Gamma}\right\}$.

Case 2.1. $I=I_{\Gamma}=\{1, . ., N\}$.

Using (1) we get $E_{\Gamma}=E$ which is a contradiction to our assumption (12).

Case 2.2. $I_{\Gamma} \backslash I \neq \emptyset$.

Let $x, y \in E$ such that $\operatorname{diam}(E)=\|x-y\|$. If both $x$ and $y$ lies in $E_{\Gamma}$, then $\operatorname{diam}(E) \leq \operatorname{diam}\left(E_{\Gamma}\right)$, which contradicts our assumption (12). Hence, w.l.o.g. we can assume, that $x \in E \backslash E_{\Gamma}$. Using equation (1), an $i \in\{1, \ldots, N\}$ exists, with $x \in S_{i}(E) \backslash E_{\Gamma}$. Hence $i \in I_{\Gamma} \backslash I$ and (dependent from $x$ ) a $k_{i} \geq 2$ and $\sigma(i)=\left(\sigma_{1}(i), . ., \sigma_{k_{i}}(i)\right) \in\{1, . ., N\}^{k_{i}}$ exists, with

(i) $x \in S_{\sigma_{1}(i)} \circ \cdots \circ S_{\sigma_{k_{i}}(i)}(E)$ and $\sigma_{1}(i)=i$,

(ii) $S_{\sigma_{1}(i)} \circ \cdots \circ S_{\sigma_{k_{i}}(i)}(E) \cap E_{\Gamma}=\emptyset$ and

(iii) $S_{\sigma_{1}(i)} \circ \cdots \circ S_{\sigma_{k_{i}-1}(i)}(E) \cap E_{\Gamma} \neq \emptyset$. 
Fix $j \in\{1, . ., N\}$ with $y \in S_{j}(E)$. If $j \in I_{\Gamma} \backslash I$, then we proceed as above and choose $k_{j} \geq 2$ and $\sigma(j) \in\{1, . ., N\}^{k_{j}}$ with properties (i)-(iii). Otherwise, i.e. in case of $j \in I$, we define $k_{j}=2$ and choose $\sigma(j)=\left(\sigma_{1}(j), \sigma_{2}(j)\right) \in$ $\{1, . ., N\}^{2}$ such that $\sigma_{1}(j)=j$ and $y \in S_{\sigma_{1}(j)} \circ S_{\sigma_{2}(j)}(E)$. Thus we deduce the existence of $x^{\prime} \in S_{\sigma(i)^{-}}(E) \cap E_{\Gamma}$ resp. $y^{\prime} \in S_{\sigma(j)^{-}}(E) \cap E_{\Gamma}$, with

$$
\begin{aligned}
\operatorname{diam}(E) & \leq\left\|x-x^{\prime}\right\|+\left\|x^{\prime}-y^{\prime}\right\|+\left\|y^{\prime}-y\right\| \\
& \leq \operatorname{diam}\left(S_{\sigma(i)^{-}}(E)\right)+\operatorname{diam}\left(E_{\Gamma}\right)+\operatorname{diam}\left(S_{\sigma(j)^{-}}(E)\right) \\
& \leq \operatorname{diam}\left(E_{\Gamma}\right)+2 \operatorname{diam}(E) \max \left(c^{k_{i}-1}, c^{k_{j}-1}\right) \\
& =\operatorname{diam}\left(E_{\Gamma}\right)+2 \operatorname{diam}(E) c^{\min \left(k_{i}, k_{j}\right)-1} .
\end{aligned}
$$

On the other hand we deduce

$$
\sum_{\sigma \in \Gamma} c_{\sigma}^{D}= \begin{cases}1-c^{D k_{i}} & , \text { if } j \in I \\ 1-\left(c^{D k_{i}}+c^{D k_{j}}\right) & , \text { if } j \in I_{\Gamma} \backslash I,\end{cases}
$$

Due to $k_{i} \geq 2$ and $k_{j}=2$, if $j \in I$ we obtain $\sum_{\sigma \in \Gamma} c_{\sigma}^{D} \leq 1-c^{D \min \left(k_{i}, k_{j}\right)}$. Thus we get with $k=\min \left(k_{i}, k_{j}\right) \geq 2$ and lemma 3.2 that

$$
\frac{\operatorname{diam}\left(E_{\Gamma}\right)^{D}}{\sum_{\sigma \in \Gamma} c_{\sigma}^{D}} \geq \operatorname{diam}(E)^{D} \frac{\left(1-2 c^{k-1}\right)^{D}}{1-c^{k D}}>\operatorname{diam}(E)^{D},
$$

which contradicts 11 .

Based on theorem 2.5 it is now straightforward to prove the second main result.

Proof of theorem 2.7.

Consider a parametrised UIFS with contracting factor $c$ and fixpoint set $\omega$ independent of $c$. Using $0<D=\frac{-\log (N)}{\log (c)}$ and $(6)$ we deduce in case of $N \geq 3$ that

$$
\Delta \leq\left(\frac{\operatorname{diam}(\omega)}{d_{\min }(\omega)}\right)^{D} \frac{N-1}{N} .
$$

The right hand side of 18 tends to $\left.\frac{N-1}{N} \in\right] 0,1[$ as $c$ tends to zero. Hence, fix $c_{1}>0$, such that $\left(\frac{\operatorname{diam}(\omega)}{d_{\min }(\omega)}\right)^{D} \frac{N-1}{N} \leq 1-\frac{1}{2 N}$, for every $\left.\left.c \in\right] 0, c_{1}\right]$. By 18 we have $\Delta(c) \leq 1-\frac{1}{2 N}<1$ for every $\left.c \in\right] 0, c_{1}$ ]. Now define $c_{0}$ as in (7) with $\Delta=\Delta\left(c_{1}\right)$. Because $c_{1}$ does only depend on $\omega$, also $c_{0}$ is fully determined by $\omega$. If the contracting factor $c$ is smaller than $\min \left(c_{0}, c_{1}\right)$, then the assertion follows immediately from theorem 2.5 . 


\section{Examples}

In this section we will give four examples, showing the applicability of our results. Moreover we compare our results with research already done in this area. (cf. remark 4.4). For our first example we need the following two technical results.

Lemma 4.1. Let $N \geq 5$ and

$$
\left[2, \frac{N}{2}+1\right] \ni x \rightarrow f(x)=\frac{x}{N \sin \left(\frac{\pi}{N}(x-1)\right)} .
$$

Then, $\max \left\{f(x): x \in\left[2, \frac{N}{2}+1\right]\right\}<\frac{N-1}{N}$.

Proof. $f$ is differentiable on $] 2, \frac{N}{2}+1[$ with

$$
f^{\prime}(x)=\frac{N \sin \left(\frac{\pi}{N}(x-1)\right)-\pi x \cos \left(\frac{\pi}{N}(x-1)\right)}{N^{2} \sin ^{2}\left(\frac{\pi}{N}(x-1)\right)} .
$$

With $z=\frac{\pi}{N}(x-1)$ let

$$
g(z)=\sin (z)-\left(z+\frac{\pi}{N}\right) \cos (z) .
$$

Observe that $g\left(\frac{\pi}{2}\right)=1$ and $g\left(\frac{\pi}{N}\right)<\frac{\pi}{N}\left(1-2 \cos \left(\frac{\pi}{N}\right)\right)<\frac{\pi}{N}\left(1-2 \cos \left(\frac{\pi}{4}\right)\right)<0$. Due to $g(z)=\cos (z)\left(\tan (z)-\left(z+\frac{\pi}{N}\right)\right)$ for $z \in\left[\frac{\pi}{N}, \frac{\pi}{2}[\right.$ we deduce with $\tan ^{\prime}(z)>1$ for every $\left.z \in\right] 0, \frac{\pi}{2}\left[\right.$ that a $\left.z_{0} \in\right] \frac{\pi}{N}, \frac{\pi}{2}[$ exists, with

(a) $g\left(z_{0}\right)=0$

(b) $g(z)<0$ for every $z \in\left[\frac{\pi}{N}, z_{0}[\right.$

(c) $g(z)>0$ for every $\left.z \in] z_{0}, \frac{\pi}{2}\right]$

Using (a)-(c) and (19) we deduce, that $f$ attains its maximum in $x=2$ or $x=\frac{N}{2}+1$. Hence, together with $\sin (x) \geq x-x^{3} / 6$ for every $x \geq 0$ we obtain

$$
\begin{aligned}
& \max \{f(x): x \in[2, N / 2+1]\} \\
= & \max \left(\frac{\frac{2}{N}}{\sin \left(\frac{\pi}{N}\right)}, \frac{\frac{N}{2}+1}{N}\right) \\
\leq & \max \left(\frac{2}{\pi-\frac{1}{N^{2}} \frac{\pi^{3}}{6}}, \frac{1}{2}+\frac{1}{N}\right)<\frac{4}{5} \leq \frac{N-1}{N} .
\end{aligned}
$$


Lemma 4.2. Let $N \geq 3$. Then $\frac{1}{4 N} \sin \left(\frac{\pi}{N}\right)>\frac{1}{2 N^{2}}$.

Proof. If $N=3$ resp. $N=4$, then the assertion follows by direct calculations. If $N=5$, we get $\frac{1}{4 N} \sin \left(\frac{\pi}{N}\right)=\frac{1}{20} \sin \left(\frac{\pi}{5}\right)>\frac{1}{20} \sin \left(\frac{\pi}{6}\right)=\frac{1}{40}>\frac{1}{50}=\frac{1}{2 N^{2}}$. Now let $N \geq 6$. Using the relation $\sin (x) \geq \frac{3}{\pi} x$ for every $x \in\left[0, \frac{\pi}{6}\right]$ we obtain $\frac{1}{4 N} \sin \left(\frac{\pi}{N}\right) \geq \frac{1}{4 N} \frac{3}{N}=\frac{3}{4} \frac{1}{N^{2}}>\frac{1}{2 N^{2}}$.

Example 4.3 (regular $N$-gon).

Let $N \geq 3$ and $\omega=\left\{x_{1}, . ., x_{N}\right\}$ be a subset of the circle

$$
C=\left\{x \in \mathbb{R}^{2}:\|x\|=1 / 2\right\} .
$$

Assume, that $\omega$ represents the vertices of a regular $N$-gon. We consider a UIFS, where each similitude $S_{i}$ has the fixpoint $x_{i}$ and contracting ratio $c \in] 0, \frac{1}{N}\left[\right.$ independent of $i \in\{1, . ., N\}$. Every $S_{i}$ may also have a rotation part, i.e. for $x \in \mathbb{R}^{d}$ we have

$$
S_{i}(x)=c O_{i}\left(x-x_{i}\right)+x_{i}
$$

with an orthonormal mapping $O_{i}: \mathbb{R}^{2} \rightarrow \mathbb{R}^{2}$. According to remark 2.6 an $I \subset\{1, . ., N\}$ exists, with $2 \leq \operatorname{card}(I) \leq N-1$ and

$$
\Delta \leq \Delta^{\prime}=\frac{\operatorname{card}(I)}{N} \frac{\operatorname{diam}(\omega)}{\operatorname{diam}\left(\omega_{I}\right)} .
$$

For $0 \leq \varphi_{1}<\varphi_{2}<2 \pi$ let

$$
C_{\left[\varphi_{1}, \varphi_{2}[\right.}=\left\{\left(\frac{1}{2} \cos (\varphi), \frac{1}{2} \sin (\varphi)\right): \varphi \in\left[\varphi_{1}, \varphi_{2}[\} .\right.\right.
$$

Thus, $C=C_{[0,2 \pi[}$. We say, that $\omega_{I}$ is dense-packed, if $\varphi_{1}(I), \varphi_{2}(I) \in[0,2 \pi[$ exist, with

(i) $0 \leq \varphi_{1}(I)<\varphi_{2}(I)<2 \pi$,

(ii) $\omega_{I} \subset C_{\left[\varphi_{1}(I), \varphi_{2}(I)[\right.}$ and

(iii) $\omega \backslash \omega_{I} \subset C \backslash C_{\left[\varphi_{1}(I), \varphi_{2}(I)[\right.}$.

W.l.o.g. we can assume, that $\omega_{I}$ is dense-packed, because otherwise we can transform it into a dense-packed set without increasing the diameter. To see this, let $x, y \in \omega_{I}$ with $\|x-y\|=\operatorname{diam}\left(\omega_{I}\right)$. The case $\operatorname{diam}\left(\omega_{I}\right)=1$ is of 
no interest. If $\operatorname{diam}\left(\omega_{I}\right)<1$, let $\varphi_{1} \in\left[0,2 \pi\left[\right.\right.$ with $x=(1 / 2)\left(\cos \left(\varphi_{1}\right), \sin \left(\varphi_{1}\right)\right)$ and $\varphi_{2} \in\left[0,2 \pi\left[\right.\right.$ with $y=(1 / 2)\left(\cos \left(\varphi_{2}\right), \sin \left(\varphi_{2}\right)\right)$. By a suitable rotation of $\omega_{I}$ about the origin we can assume w.l.o.g. that $0=\varphi_{1}<\varphi_{2}<2 \pi$. Moreover the problem remains unchanged in case of a reflection of $\omega_{I}$ with respect to the abscissa. Hence we can assume w.l.o.g. that $\varphi_{2}<\pi$. Due to $\|x-y\|=\operatorname{diam}\left(\omega_{I}\right)<1$ we deduce $\omega_{I} \subset C \backslash C_{] \varphi_{2}, 2 \pi-\varphi_{2}[}$ with

$$
\operatorname{diam}\left(\omega_{I}\right)=\operatorname{diam}\left(C \backslash C_{] \varphi_{2}, 2 \pi-\varphi_{2}[}\right) .
$$

Hence $\omega_{I}$ can be transformed into a dense-packed set $\omega_{I}^{\prime} \subset C \backslash C_{] \varphi_{2}-\delta, 2 \pi-\varphi_{2}+\delta[}$ with a $\delta \in\left[0, \varphi_{2}[\right.$. Using (22) we deduce

$$
\operatorname{diam}\left(\omega_{I}^{\prime}\right) \leq \operatorname{diam}\left(C \backslash C_{] \varphi_{2}-\delta, 2 \pi-\varphi_{2}+\delta[}\right) \leq \operatorname{diam}\left(\omega_{I}\right) .
$$

Now let $\omega_{I}$ be dense-packed. Thus we deduce

$$
\operatorname{diam}\left(\omega_{I}\right) \geq \begin{cases}\sin \left(\frac{\pi}{N}(\operatorname{card}(I)-1)\right) & , \text { if } \operatorname{card}(I) \leq \frac{N}{2}+1 \\ \operatorname{diam}(\omega) & , \text { otherwise }\end{cases}
$$

Using (21) one gets by a direct calculation, that $\Delta \leq \frac{N-1}{N}$ for $N=3$ and $N=4$. In case of $N \geq 5$, the combination of (21) and (23) together with $\operatorname{diam}(\omega) \leq 1$ implies

$$
\Delta \leq \begin{cases}\frac{\operatorname{card}(I)}{N}\left(\sin \left(\frac{\pi}{N}(\operatorname{card}(I)-1)\right)\right)^{-1} & , \text { if } \operatorname{card}(I) \leq \frac{N}{2}+1 \\ \frac{\operatorname{card}(I)}{N} & , \text { otherwise. }\end{cases}
$$

With lemma 4.1 we obtain $\Delta \leq \frac{N-1}{N}$. Hence, $\Delta \leq \frac{N-1}{N}$ for every $N \geq 3$. With $d_{\min }(\omega)=\sin \left(\frac{\pi}{N}\right)$ and lemma 4.2 the upper bound for $c$, required in theorem 2.5, is greater or equal to

$$
\min \left(\left(2 N^{2}\right)^{-1}, \frac{1}{4} \sin \left(\frac{\pi}{N}\right)\left(1-\frac{N-1}{N}\right)\right)=\left(2 N^{2}\right)^{-1} .
$$

Hence, if $c<\left(2 N^{2}\right)^{-1}$ we can apply theorem 2.5 and obtain $\mathcal{H}^{D}(E)=$ $\operatorname{diam}(E)^{D}$.

Remark 4.4. For the one-dimensional dyadic homogeneous Cantor set, i.e. $d=1$ and $N=2$ with uniform contracting factor $\left.c \in] 0, \frac{1}{2}\right]$ it is well-known, that $\mathcal{H}^{D}(E)=\operatorname{diam}(E)^{D}$. Our approach (cf. theorem 2.5) is restricted to $c \in] 0, \frac{1}{8}[$ in this case. If we consider the (general) Sierpinksi gasket, i.e. 
$d=2$ and $\operatorname{card}(\omega)=N=3$, where $\omega$ consists of the vertices of an equilateral triangle, then we obtain from example 4.3 that $\mathcal{H}^{D}(E)=\operatorname{diam}(E)^{D}$, provided the uniform contracting factor $c$ lies in $] 0, \frac{1}{18}[$. By other methods, more adapted to the special geometry of the Sierpinski gasket, it was shown by several authors (cf. [5, 26]) that this identity is also true, if $\left.c \in] \frac{1}{4}, \frac{1}{3}\right]$. But they needed the restriction, that every similitude of the UIFS does not contain a rotation, i.e. in (20) for every $i \in\{1, . ., N\}$ the mapping $O_{i}$ is the identical mapping. In our approach this restriction is not necessary. Also the Sierpinski carpet, i.e. the case $N=4$ in example 4.3 has been investigated. It was first shown by Zhu and Lou [28], that $\mathcal{H}^{D}(E)=\operatorname{diam}(E)^{D}$ holds, if $c \in] 0,1 / 4]$ and the UIFS does not contain any rotation parts. See also [4, 21] for a proof. Our results from example 4.3 show this identity only for $c \in] 0,1 / 32[$, but they allow rotation in the UIFS. In case of $N=2 m$ with a positive integer $m$, it was shown by $W u$ [20], that $\mathcal{H}^{D}(E)=\operatorname{diam}(E)^{D}$ is true, if the contracting factor $c$ is smaller or equal to $\frac{1}{N}$. Again, our results are restricted to $c \in] 0, \frac{1}{2 N^{2}}[$ but allow rotation parts in the UIFS. For odd numbers $N \geq 5$, the results in example 4.3 seem to be new.

In context with two-dimensional UIFS it is also interesting to study the applicability of theorem 2.5 for the following example.

Example 4.5 (modified Vicsek fractal). Chen and Yang [3] studied the Hausdorff measure of another two-dimensional fractal set. They considered the UIFS consisting of the fixed points $\omega=$ $\left\{x_{1}, . ., x_{5}\right\}$ with $x_{1}=(0,0), x_{2}=(1,0), x_{3}=(1,1), x_{4}=(0,1)$ and $x_{5}=$ $(1 / 2,1 / 2)$. If the UIFS contains no rotation parts and the contracting factor is lower or equal to $\frac{1}{N}$, they have proved, that $\mathcal{H}^{D}(E)=\operatorname{diam}(E)^{D}$. An easy calculation shows $\Delta^{\prime}=\frac{3 \sqrt{2}}{5}<1$, resp. $\quad d_{\min }(\omega)=\frac{1}{2} \sqrt{2}$ and $\operatorname{diam}(\omega)=\sqrt{2}$. Thus we deduce from theorem 2.5, that above equality for $\mathcal{H}^{D}(E)$ holds, if $0<c<\frac{1}{8}\left(1-\frac{3 \sqrt{2}}{5}\right)=0.0189$.. which is, in this respect, weaker than the result of Chen and Yang, but we allow, that the UIFS can have rotation parts.

Our third example illustrates, that theorem 2.7 does not hold, if the set $\omega$ of fixed points of the (parametrised) UIFS may also depend on the contracting parameter $c$.

Example 4.6. Let $d=2$ and $N=3$. For $x>0$ and $\alpha>0$ let $x_{1}=(0,0)$, $x_{2}=(x, 0)$ resp. $x_{3}=(0, x \sqrt{\alpha})$. With the uniform contracting factor $c=$ 
$\frac{1}{(1+\alpha)}$ we consider the UIFS consisting of the similitudes $S_{i}(\cdot)=c\left(\cdot-x_{i}\right)+x_{i}$ for $i \in\{1,2,3\}$. Let $\Gamma=\{1,2\}$. Clearly, $\operatorname{diam}(\omega)=x \sqrt{1+\alpha}$. We obtain

$$
\begin{aligned}
\sum_{i \in \Gamma} \operatorname{diam}\left(S_{i}(E)\right)^{D} & =2 c^{D} \operatorname{diam}(E)^{D} \\
& \geq 2 c^{D} \operatorname{diam}(\omega)^{D}=2 c^{D}(x \sqrt{1+\alpha})^{D} .
\end{aligned}
$$

On the other hand, Lemma 3.1 (a) implies

$$
\operatorname{diam}\left(E_{\Gamma}\right)^{D} \leq(x+2 c \operatorname{diam}(E))^{D}<\left(x+\frac{2 c}{1-2 c} x \sqrt{1+\alpha}\right)^{D} .
$$

From (24), 25) and $c^{D}=\frac{1}{3}$ we deduce

$$
\frac{\operatorname{diam}\left(E_{\Gamma}\right)^{D}}{\sum_{i \in \Gamma} \operatorname{diam}\left(S_{i}(E)\right)^{D}}<\frac{3}{2}\left(\frac{1}{\sqrt{1+\alpha}}+\frac{2 c}{1-2 c}\right)^{D} .
$$

Let us assume, that $\alpha \geq 3$. By $c=\frac{1}{1+\alpha}$ we get $\frac{2 c}{1-2 c} \leq 4 c=\frac{4}{1+\alpha}$. Hence, the right hand side of (26) is smaller or equal to

$$
\frac{3}{2}\left(\frac{1}{\sqrt{1+\alpha}}+\frac{4}{1+\alpha}\right)^{\frac{\log (3)}{\log (1+\alpha)}}=\frac{\sqrt{3}}{2}\left(1+\frac{4}{\sqrt{1+\alpha}}\right)^{\frac{\log (3)}{\log (1+\alpha)}}
$$

which tends to $\frac{\sqrt{3}}{2}<1$ as $\alpha \rightarrow \infty$. Especially if $\alpha \geq 40$, it is easy to calculate, that the right hand side of (27) is smaller than 1, yielding with (26) that

$$
\frac{\operatorname{diam}\left(E_{\Gamma}\right)^{D}}{\sum_{i \in \Gamma} \operatorname{diam}\left(S_{i}(E)\right)^{D}}<1
$$

Using $\Gamma=\{1,2\} \subset\{1, \ldots, N\}^{k_{0}}$ with $k_{0}$ as in (3) we deduce with theorem 2.7 (c) that

$$
\operatorname{diam}(E)^{D}>\frac{\operatorname{diam}\left(E_{\Gamma}\right)^{D}}{\sum_{i \in \Gamma} c_{i}^{D}} \geq \mathcal{H}^{D}(E)
$$

Moreover,

$$
\Delta \geq \frac{2}{3}(\sqrt{1+\alpha})^{\frac{\log (3)}{\log (1+\alpha)}}=\frac{2}{\sqrt{3}}>1
$$


Remark 4.7. Example 4.6 shows, that $\Delta<1$ cannot be dropped in theorem 2.5, i.e. theorem 2.5 becomes wrong, if we only require $c_{0}=\left(2 N^{2}\right)^{-1}$. If $\Delta<1$ holds, the upper bound for the contracting factor, required in theorem 2.5. will certainly not be optimal at all. It is natural to ask, if we can weaken or drop this boundary condition and require only $\Delta<1$. Unfortunately we cannot do so. This follows from the work for the Sierpinski gasket (cf. [2] and the references therein). Using resp. slightly adapting the methods in [2], it is easy to show, that $\mathcal{H}^{D}(E)<(\operatorname{diam}(E))^{D}$ and $(S S C)$ is satisfied, if the contracting factor $c$ is smaller but close enough to $\frac{1}{2}$. Clearly, $\Delta=\frac{2}{3}<1$ is still satisfied in this situation.

We finish this section with a higher dimensional example.

Example 4.8. Let $d \geq 2$ and $\omega$ be the vertices of the $d$-dimensional unit cube $[0,1]^{d}$. Consider a parametrised UIFS with $\omega$ as fixpoint set and contracting factor $c$. By theorem 2.7 a constant $b_{0}=b_{0}(\omega)>0$ exists, such that $\mathcal{H}^{D}(E)=\operatorname{diam}(E)^{D}$ if $\left.c \in\right] 0, b_{0}[$. If the UIFS does not contain any rotation parts, it was shown by Zhou et.al. [25] that $b_{0} \geq 2^{-d}$.

\section{Concluding remarks}

Recall $\mathcal{E}$ as defined in (5). It would be of general interest, which sets $\mathcal{E}$ consists of, resp. which cardinality $\mathcal{E}$ has. A possible answer to this question will likely depend on the special structure of the IFS, especially if it contains rotation parts. Moreover one could ask, what implications the equation

$$
\mathcal{H}^{D}(E)=\operatorname{diam}(E)^{D}
$$

does have. Note, that this equality does not imply condition (SSC). If we e.g. consider the unit interval $[0,1]$ as the invariant set of the UIFS with

$S_{1}(x)=\frac{1}{2} x$ and $S_{2}(x)=\frac{1}{2} x+\frac{1}{2}$ for every $x \in \mathbb{R}$, then this UIFS satisfies 28) but not condition (SSC). It would be interesting to know, if (28) implies, that $\mathcal{E}$ has finite cardinality.

The applicability of theorem 2.5 relies on the fact $\Delta<1$. This is ensured by $\Delta^{\prime}<1$ (cf. remark 2.6). In practice, to verify $\Delta^{\prime}<1$ one has to evaluate all appearing combinations of subsets $\omega_{I}$ of $\omega$ with $2 \leq \operatorname{card}(I) \leq N-1$. Thus we have to compute

$$
\sum_{k=2}^{N-1}\left(\begin{array}{l}
N \\
k
\end{array}\right)=2^{N}-N-2
$$


combinations. Clearly, this becomes quite hard if $N$ becomes large, unless $\omega$ has a specific geometric structure (cf. example 4.3). But also in highly symmetric cases it could be difficult to check, if $\Delta^{\prime}<1$. E.g. this is the case in example 4.8 .

Let us consider a parametrised UIFS. Note that the attractor $E$ and the Hausdorff dimension $D$ depends on the contracting factor $c$. Let

$$
\left.\left.M_{1}=\{c \in] 0,1\left[\text { UIFS satisfies SSC for every } c^{\prime} \in\right] 0, c\right]\right\}
$$

and let

$$
\left.\left.\emptyset \neq M_{2}=\left\{c \in M_{1}: \mathcal{H}^{D}(E)=\operatorname{diam}(E)^{D} \text { for every } c^{\prime} \in\right] 0, c\right]\right\} .
$$

In view of remark 4.7 it is natural to ask about the determination of $d_{0}=$ $\sup M_{2}$. Note that $d_{0} \in M_{2}$ by reasons of continuity (cf. [13]). As already stated above, also here a possible answer will likely depend on the geometry of the UIFS.

Finally it also remains open to find UIFS, if existing, satisfying $(28)$ and $D>1$.

\section{References}

[1] E. Ayer, R.S. Strichartz, Exact Hausdorff measure and intervals of maximum density for Cantor sets, Trans. Am. Math. Soc. 351 (1999), 37253741 .

[2] J. Baoguo, Bounds of Hausdorff measure of the Sierpinski gasket, J. Math. Anal. Appl. 330 (2007), 1016-1024.

[3] D. Chen, X. Yang, Hausdorff measures of a class of Sierpinski carpets, Anal. Theory Appl. 20 (2004), 167-174.

[4] Z. Cui, Y. Yang, C. Wang, The Hausdorff measure of a generalized Sierpinski carpet, Chin. Q. J. Math. 18 (2003), 57-67.

[5] Z. Gui, G. Ma, The Hausdorff measure of a kind of Sierpinski gasket, J. Henan Norm. Univ., Nat. Sci. 29 (2001), 93-94.

[6] F. Hausdorff, Dimension und äußeres Maß, Math. Ann. 79 (1918), 157179. 
[7] W.H. He, J. Luo, Z.L. Zhou, Hausdorff measure and isodiametric inequalities, Acta Math. Sinica (Chin. Ser.) 48 (2005), 939-946.

[8] S. Hua, Dimensions for generalized self-similar sets, Acta Math. Appl. Sinica (Chin. Ser.) 17 (1994), 551-558.

[9] S. Hua, Z.Y. Wen, H. Rao, J. Wu, On the structures and dimensions of Moran sets, Sci. China Ser. A 8 (2000), 836-852.

[10] R. Huojun, S. Weiyi, An approximation method to estimate the Hausdorff measure of the Sierpinski gasket, J. Anal. Theory Appl. 20 (2004), $158-166$.

[11] J.E. Hutchinson, Fractals and self-similarity, Indiana Univ. Math. J. 30 (1981), 713-747.

[12] M. Llorente, M. Morán, Self-similar sets with optimal coverings and packings, J. Math. Anal. Appl. 334 (2007), 1088-1095.

[13] J. Luo, Z.L. Zhou, Hausdorff measure of self-similar sets and continuity, Acta Math. Sinica (Chin. Ser.) 46 (2003), 457-462.

[14] C. Ma, Hausdorff measure of linear Cantor set, Wuhan Univ. J. Nat. Sci. 9 (2004), 135-138.

[15] J. Marion, Mesure de Hausdorff d'un fractal à similitude interne, Ann. Sci. Math. Quebec 10 (1986), 51-84.

[16] J. Marion, Mesures de Hausdorff d'ensembles fractals, Ann. Sci. Math. Quebec 11 (1987), 111-132.

[17] P. Moran, Additive functions of intervals and Hausdorff measure, Proc. Camb. Phil. Soc. 42 (1946), 15-23.

[18] C.Q. Qu, R. Rao, W.Y. Su, Hausdorff Measure of Homogeneous Cantor Set, Acta Math. Sin. (Engl. Ser.) 17 (2001), 15-20.

[19] C.Q. Qu, Z.L. Zhou, Hausdorff measures for a class of homogeneous Moran sets, Nonlinear Anal. Theory Methods Appl. 65 (2006), 442-447.

[20] J. Wu, The Hausdorff measure of a class of Sierpinski carpets based on polygons with even edges, J. Xiamen Univ. Nat. Sci. 41 (2002), 404-408. 
[21] Y. Xiong, J. Zhou, The Hausdorff measure of a class of Sierpinski carpets, J. Math. Anal. Appl. 305 (2005), 121-129.

[22] S. Xu, W. Su, Z.L. Zhou, On the exact Hausdorff measure of a class of self-similar sets satisfying open set condition Anal. Theory Appl. 24 (2008), 93-100.

[23] C. Zeng, D. Yuan, The Hausdorff measure of $m$ non-uniform Cantor Set, Far East J. Math. Sci. 22 (2006), 239-248.

[24] Z. Zhou, L. Feng, Twelve open problems on the exact value of the Hausdorff measure and on topological entropy: a brief survey of recent results, Nonlinearity 17 (2004), 493-502.

[25] Z. Zhou, M. Wu, Y. Zhao, The Hausdorff measure of a class of generalized Sierpinski sponges, Chinese Ann. Math. Ser. A 22 (2001), 57-64.

[26] Z.Y. Zhou, Z.G. Yu, The Exact Hausdorff Measure of General Sierpinski Gasket With Similitude Ratio a $\in[1 / 4,1 / 3)$, Natur Sci. J. Xiangtan Univ. 29 (2007), 5-9.

[27] Z. Zhou, J. Le, Some problems on fractal geometry and dynamical systems, J. Eng. Math. Xi'an 23 (2006), 761-766.

[28] Y. Zhu, J. Lou, The Hausdorff measure of generalized Sierpinski carpets, Approx. Theory Appl. (N.S.) 16 (2000), 13-18. 